\title{
Objective 3-D Breast Symmetry Evaluation for Clinical Application using Three-Dimensional Laser Light Surface Imaging
}

\author{
Fee ARMBRECHT*a, Maximilian EDER ${ }^{\mathrm{a}}$, Stefan RAITH ${ }^{\mathrm{a}}$, Alexander VOLF ${ }^{\mathrm{a}}, \mathrm{A}$. SWOBODNIK ${ }^{\mathrm{a}}$, \\ A.-K. PAPE ${ }^{a}$, T. SCHUSTER ${ }^{b}$, Nikolaos. A. PAPADOPULOS ${ }^{a}$, H.-G. MACHENS ${ }^{a}$, \\ and Laszlo KOVACS ${ }^{a}$ \\ ${ }^{a}$ Research Group Computer Aided Plastic Surgery - CAPS, \\ Clinic for Plastic Surgery and Hand Surgery, Technical University Munich, Germany; \\ ${ }^{\mathrm{b}}$ Institute of Medical Statistics and Epidemiology, Technical University Munich, Germany
}

\begin{abstract}
Background and Objectives: Currently differences in breast shape and size to evaluate breast symmetry are subjectively estimated by surgeons relying on visual assessments, experience and judgment. A variety of methods have been described to assess breast symmetry, but no technique is generally accepted. 3-D surface imaging provides a new alternative to objectively quantify volume, shape, contour, surface and symmetry of the breast region. The purpose of this study is to standardize a 3-D breast symmetry evaluation protocol regarding improved precision and a more relevant clinical application tool for preoperative planning and evaluation of the postoperative outcome using 3-D laser light surface scanner.
\end{abstract}

Study Design/Materials and Methods: According to specific anatomical landmarks of the breast region a 3-D evaluation protocol was designed to quantify symmetry in all the four quadrants of the breast. Three independent observers standardized the 3-D evaluation protocol using two symmetrical dummy models with different breast sizes $(n=60)$, examined its applicability with 10 test subjects $(n=300)$ and applied it to 3 clinical patients (1 breast augmentation, 1 breast reduction and 1 breast reconstruction).

Results: Intra- and inter-observer differences regarding the precision (reproducibility) of the method were not statistically significant during standardization using dummies nor during test subject applications. The precision of the breast symmetry evaluation, expressed as the coefficient of variation, was $3.33+/-0.13$ for the test subjects and therefore significantly higher than the precision for the dummy models, $1.66+/-0,14$ for dummy $A$ and $1.91+/-0.11$ for dummy $B(p<0.001)$. The breast symmetry evaluation protocol quantifies existing pre- and postoperative differences regarding volume, surface, shape, projection, contour and symmetry and provides more objective documentation for clinical application.

Conclusions: The 3-D breast symmetry evaluation protocol is an easily applicable, observer independent and rapidly learnable method which is sufficiently precise and observer independent. Breast symmetry evaluation in three-dimensions is a useful and valuable clinical tool that enables surgeons to quantify and objectively visualise existing breast asymmetry for planning purposes, postoperative outcome research analysis and could help to prevent or correct postoperative asymmetries in terms of a postoperative quality assurance.

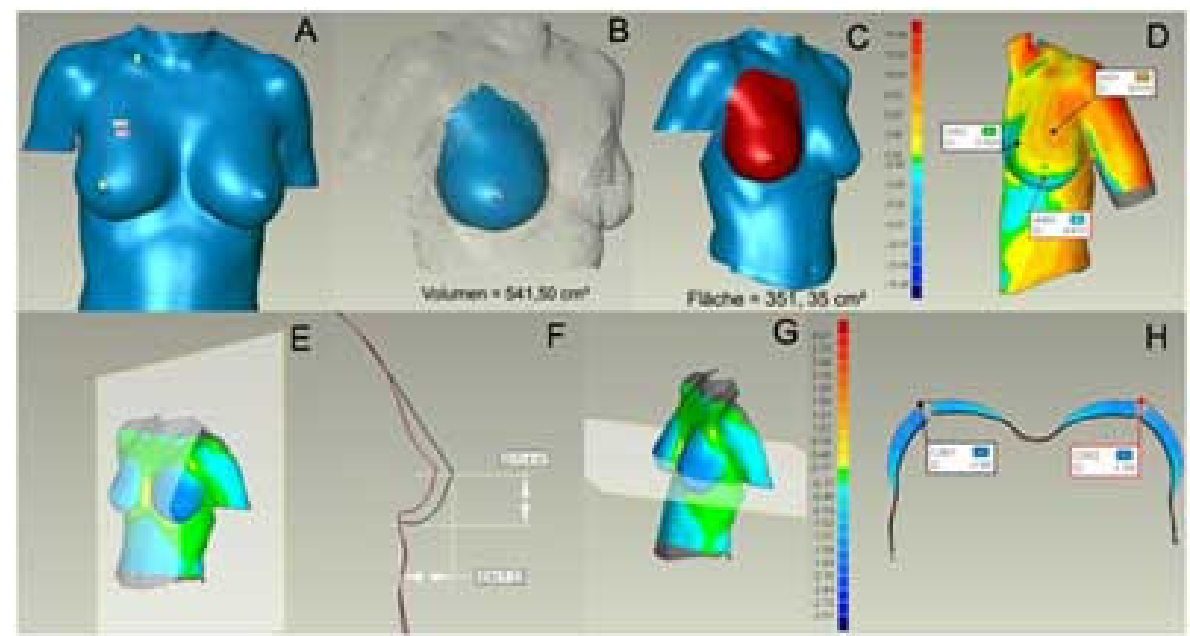

*fee.armbrecht@Irz.tum.de 